Facsimile Price $\$$

Microfilm Price \$

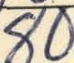

trailable from the

Office of Technical Services

Department of Commerce

Washington 25, D. C.
ACCELERATOR DEPARTMENT

EDC -45
Internal Report

BROOKHAVEN NATIONAL IABORATORY

Associated Universities, Inc. Upton, L.I., N.Y.

\title{
ACCELERATION OF POLARIZED PROTONS TO RELATIVISTIC ENERGIES*
}

\section{E.D. Courant \\ Yale University and Brookhaven National Laboratory \\ January 22, 1962}

We have just heard about progress in the production of beams of polarized protons and deuterons. It would clearly be desirable to accelerate such beams to high energies in order to obtain data on the spin dependence of various high energy reactions. Therefore one may investigate whether, when protons are accelerated in existing machines such as a Cosmotron, Alternating Gradient Synchrotron, etc., the polarization of a bunch of protons can be preserved.

Linear accelerators seem to present no great problems. At Harwe11, polarized protons have been successfully accelerated to $40 \mathrm{Mev}$, and any 1 inear accelerators that are used as injectors into large circular machines may also be expected to preserve the polarization of protons.

The situation is different in the case of circular accelerators, in which the protons are made to go in circles by a magnetic guide field. The spin will precess in this same guide field, and its axis of precession will be affected by whatever perturbing effects are present.

These effects have been investigated by a number of people, including Froissart and Stora ${ }^{1}$ at Saclay, Cohen ${ }^{2}$ Argonne, and Lobkowicz and Thorndike ${ }^{3}$ at Rochester. Thus I can make no claim to originality here. But while the people I just mentioned have concentrated on the specific accelerators at their particular laboratories, I should like to investigate the problem of the interaction of orbit dynamics and particle polarization in general, to see what characteristics of any particular accelerator affect the polarization.

* Talk being presented at American Physical Society Meeting, January 25, 1962 


\section{DISCLAIMER}

This report was prepared as an account of work sponsored by an agency of the United States Government. Neither the United States Government nor any agency Thereof, nor any of their employees, makes any warranty, express or implied, or assumes any legal liability or responsibility for the accuracy, completeness, or usefulness of any information, apparatus, product, or process disclosed, or represents that its use would not infringe privately owned rights. Reference herein to any specific commercial product, process, or service by trade name, trademark, manufacturer, or otherwise does not necessarily constitute or imply its endorsement, recommendation, or favoring by the United States Government or any agency thereof. The views and opinions of authors expressed herein do not necessarily state or reflect those of the United States Government or any agency thereof. 


\section{DISCLAIMER}

Portions of this document may be illegible in electronic image products. Images are produced from the best available original document. 
The equation of motion of the spin vector of a charged particle in a magnetic field has been formulated by Bargmann, Mịchel and Telegdi ${ }^{4}$ using classical relativistic electrodynamics. The use of classical mechanics is justified because we are dealing with the mean value or expectation value of the spin of a large ensemble of particles, and expectation values obey classical equations of motion. Bargmann, Michel and Telegdis's equation may be written in the form

$$
\frac{\mathrm{d} \vec{\sigma}}{\mathrm{d} s}=\vec{\sigma} \times\left[(1+\gamma G) \frac{\vec{B}}{\mathrm{~B} p}+(1+G) \frac{\overrightarrow{B_{n}}}{\mathrm{~B} p}\right]
$$

where $\vec{B}_{\perp}, \vec{B}_{\| 1}$ are those parts of thẹ magnet field which are perpendicular. and parallel to the direction of motion;s is the distance traveled; $\vec{\sigma}$ is the spin vector transformed back to the $C M$ system, and $G \div \frac{8}{2}-1$ is the anomalous moment coefficient. The appearance of $\gamma$ in this equation shows that the gyromagnetic ratio, or the ratio of precession frequency to revolution frequency, becomes anisotropic as the particle energy increases, and the ratio for transverse fields increases with increasing energy. This change of the ratio with energy is what causes our difficulty, for it insures that at various energies the frequency of precession equals the frequency of some component or other of the depolarizing fleld, and when that occurs, we obtain resonant depolarization.

To see this in more detail, let us consider the vertical component of polarization (assuming the main field is vertical and the particle orbit lies in a hori.* zontal plane). Normalizing $\sigma$ to a unit vector we have

$$
\frac{d \sigma}{d s}=(1+\gamma G) \frac{B_{r}}{B \rho} \sin \Psi-(1+G) \frac{B_{s}}{B p} \cos \Psi
$$


where $\Psi$ is the azimuth of precession relative to the azimuth of revolution:

$$
\frac{d \Psi}{d s}=\frac{\gamma G}{\rho}
$$

$\theta$ is the angle between the axis of polarization and the Z-axis.

Now from the equations of motion of the particle

$$
\frac{B_{r}}{B p}=\frac{d^{2} z}{d s^{2}}
$$

and from Maxwell's equations

$$
\frac{B_{s}}{B p}= \pm \frac{z}{p} \delta\left(s-s_{\text {end }}\right)
$$

1.e. a delta function at the ends of straight section.

Here $Z$ is the vertical excursion of the particle.

Thus

$$
\frac{d \sigma}{d s}=(1+\gamma G) Z^{\prime \prime} \sin \psi \pm(1+G) \frac{Z}{\rho} \delta(s-s \text { end }) \cos \psi
$$

and resonance occurs whenever $z^{\prime \prime}$ or $\pm \frac{Z}{\rho} \delta$ (s-s end)

possesses a Fourier component of the same frequency as $\Psi$,

1.e. YG times the frequency of revolution.

In these expressions $Z$ is the total vertical displacement from the median plane, including both displacements of the equilibrium orbit due to magnet imperfections and free oscillations about the equilibrium orbit. The free oscillations have a principal component of frequency $v$ times the cyclotron frequency, plus sidebands at $\mathrm{kP} \pm v$ where $\mathrm{P}$ is the number of identical periods in the magnet structure ( in AG or straight-section machine). The orbit displacements due to imperfections contain all integral multiple of the cyclotron frequency. 
Thus resonances arise for the following values of

$$
\begin{array}{ll}
\gamma G=k P \pm v & \text { (intrinsic) } \\
\gamma G=k & \text { (imperfection) }
\end{array}
$$

To estimate the effect of these we must consider the orbit dynamics of the particular accelerator.

In a cyclotron, or a synchrotron without straight sections, $P$ is infinite, so. that only the imperfection resonances arise. Lobkowicz and Thorndike have shown that the one with $k=2$ can be troublesome at about 110 Mev unless the second harmonic component of the magnetic median plane has an amplitude of less than $1 \mathrm{~cm}$. In a weak-focusing machine with straight sections, such as the Cosmotron, Bevatron or the Saclay. synchrotron (Saturne) the straight-section term dominates, with resonances at $\gamma G=4 \pm v, 8 \pm v$ etc. (four-fold periodicity). In the Argonne machine, where the focusing field is concentrated at the maznet ends, it possesses all multiples of the eighth harmonic, and the resonances $Y G=8 \pm v$ (at about 2.8 and 3.7 Bev) are strongly excited. Finally, in strongfocusing synchrotrons, such as the Brookhaven AGS, the principal resonance arises at $\gamma G=v$; at Brookhaven $(v=8.75)$ this is at $3.6 \mathrm{Bev}$.

Now how strong are these resonances? Since the frequency of precession changes with energy, the total build-up in a passage through a resonance is inversely proportional to the square root of the rate of acceleration, and is

$$
\sqrt[1]{\gamma^{\prime} G}
$$

times the build-up in one turn $\left(\gamma^{\prime}=\right.$ change in $\gamma$ per turn). Thus the focusing term leads to a maximum build-up of

$$
\Delta \theta_{f}=\frac{\pi(1+\gamma G)(\gamma G)^{2}}{\sqrt{\gamma^{\prime} G}} \frac{z_{x}}{R}
$$

while the straight-section term leads to

$$
\Delta \theta_{s}=\frac{2 \pi(1+G) v}{\sqrt{\gamma^{\prime} G}}-\frac{L}{2 \pi R} \frac{z_{r}}{R}
$$


where $Z_{r}$ is the resonant component (of frequency $\gamma G$ ) of the vertical motion; $i / 2 \pi R$ is the fraction of the circumference occupied by straight sections. Each of these should be multiplied by the cosine of a random phase angle.

The following shows numerical values of the depolarization for $1 \mathrm{~cm}$ amplitude of oscillation:

Machine

Cosmotron

or Saturne

Princeton-Penn

Argonne ZGS

300 Bev AGS

$$
\text { YG }
$$

$$
4-v=3.1
$$$$
4+v=4.9
$$$$
8-v=7.1
$$

$$
8-v=7.1
$$$$
8+v=8.9
$$

$$
\begin{aligned}
12-v & =3.25 \\
v & =8.75
\end{aligned}
$$$$
v=30
$$

E

kin

$\begin{array}{ll}.68 \text { Bev } & 2.8 \text { radians } \\ 1.62 & 4.0 \\ 2.8 & 0.8 \\ 2.8 & 1.84 \\ 3.7 & 2.3\end{array}$

We see that the Princeton-Pennsylvania Accelerator, because of its eightfold symmetry, has its lowest intrinsic resonance only just below lts peak energy, while all the other accelerators enumerated here run into resonances at energies well below their design peaks.

These figures are discouragingly large, and yet not so large as to appear hopeless. What can be done? In the first place, one may attempt to inject an exceedingly well-collimated beam of protons making oscillations of amplitude well below $1 \mathrm{~cm}$. This reduces the depolarization angles in proportion to the amplitude. Furthermore, as suggested by Cohen, one may energize special quadrupoles along the orbit in such a way as to jump across the resonances by changing $v$ rapidly just as each resonance 18 approached. Cohen has computed the effects of 
such a program for the Argonne ZGS, and found that the depolarization could be reduced by an order of magnitude. This is obviously difficult but not necessarily impossible.

Imperfection resonances are not affected by such tricks. Numerically, they are worse for harmonic orders near $v$, since the orbit displacements have their largest components near $v$. For the Brookhaven AGS', with $k=9$, we now have an orbit that probably contains about $\frac{1}{2} \mathrm{~cm}$ of ninth harmonic; with a very careful aligning job one might conceivably reduce this to, say $1 \mathrm{~mm}$, Even then the depolarization just at that one resonance would still be more than one radian.

Finally, let me talk about the possiblity of accelerating other particles than protons. The electron has a very small anomalous moment; $G=\frac{\alpha}{2 \pi}=1.16 \times 10^{-3}$. Thus in the Cambridge Electron Accelerator $(v=6.4)$ the principal resonance occurs at $2.8 \mathrm{Bev}$, while the first imperfection resonance occurs at $\gamma G=1$, or $E=440 \mathrm{Mev}$. Thus respectable energies of polarized electrons may be attainable with such an accelerator.

If deuterons are accelerated in a proton synchrotron such as the AGS, the resonances are also moved to higher energies, since the anomalous moment of the deuteron is much smaller than that of the proton. We have $G=0.85$; thus the principal depolarization resonance in the AGS would come at

$$
z=\frac{8.75}{0.85}=10.3
$$

or at about $18 \mathrm{Bev}$. 


\section{REFERENCE\&}

1. M. Froissart and R. Stora, Nucl. Inst. 7,297 (1960)

2. D. Cohen, R.S.I. to be published

3. F. Lobkowicz and E. H. Thorndike, R.S.I., to be published

4. Bargmann, Michel and Telegdi, Phys. Rev. Letters 2, 435 (1959)

EDC:mo'n

1/22/62

Distr: $\mathrm{B} 1, \mathrm{~B} 2, \mathrm{~B} 3$ 